PROCEEDINGS OF THE

AMERICAN MATHEMATICAL SOCIETY

Volume 131, Number 12, Pages 3925-3927

S 0002-9939(03)06911-9

Article electronically published on June 30, 2003

\title{
EXACTLY $k$-TO-1 MAPS AND HEREDITARILY INDECOMPOSABLE TREE-LIKE CONTINUA
}

\author{
THOMAS E. GONZALEZ
}

(Communicated by Alan Dow)

\begin{abstract}
In 1947, W.H. Gottschalk proved that no dendrite is the continuous, exactly $k$-to- 1 image of any continuum if $k \geq 2$. Since that time, no other class of continua has been shown to have this same property. It is shown that no hereditarily indecomposable tree-like continuum is the continuous, exactly $k$-to-1 image of any continuum if $k \geq 2$.
\end{abstract}

One of the earliest results concerning exactly $k$-to- 1 maps between continua is W. H. Gottschalk's [2] result that no dendrite is the continuous exactly $k$-to- 1 image of any continuum if $k \geq 2$. Since Gottschalk's result, no other class of continua has been shown to repel exactly $k$-to- 1 functions from continua in the manner that dendrites do. It is proved that no hereditarily indecomposable tree-like continuum is the continuous exactly $k$-to- 1 image of any continuum if $k \geq 2$. This result gives more information towards a resolution of a question posed by Nadler and Ward [10] i.e., which continua are $k$-to- 1 images of continua, where $k \geq 2$ ? The result also generalizes a result of J. Heath [5] who proved that no hereditarily indecomposable tree-like continuum is a two-to-one image of a continuum. It is known that for each $k>2$ there exists a $k$-to-1 map between tree-like continua [4]. For more results concerning $k$-to-1 functions between continua, the reader is directed to a survey paper of J. Heath [7.

A space is a compact metric space, a continuum is a nonempty, compact, connected metric space, and a map is a continuous function. If $X$ and $Y$ are spaces, then a map $f$ from $X$ into $Y$ is said to be confluent if for any continuum $L$ in the image, every component of $f^{-1}(L)$ maps onto $L$.

Lemma 1. Suppose that $f$ is a confluent map onto a space $Y$ and $n$ is a positive integer such that $n \leq k$. Let $\mathcal{C}$ denote the set of all continua in $Y$ whose inverse image under $f$ has exactly $n$ components. If $\mathcal{C}$ is non-empty, then $\mathcal{C}$ has a minimal element with respect to inclusion.

Proof. It will be shown that every chain $\mathcal{L} \subset \mathcal{C}$ has a lower bound in $\mathcal{C}$, namely $\cap \mathcal{L}$. It need only be shown that $f^{-1}(\bigcap \mathcal{L})$ has $n$ components. If $L$ is an element of $\mathcal{L}$, the fact that $f$ is confluent implies that for every subcontinuum $M$ of $L$, each component of $f^{-1}(L)$ contains at least one component of $f^{-1}(M)$. If $M \in \mathcal{L}$, then $f^{-1}(M)$ and $f^{-1}(L)$ have the same number of components, in which case

Received by the editors March 1, 2001 and, in revised form, June 5, 2001.

2000 Mathematics Subject Classification. Primary 54C10.

Key words and phrases. $k$-to-1 map, hereditarily indecomposable continua, tree-like continua.

(C)2003 American Mathematical Society 
each component of $f^{-1}(L)$ contains exactly one component of $f^{-1}(M)$. Thus, the components of $f^{-1}(M)$, where $M$ 's are in $\mathcal{L}$, form $n$ decreasing chains of continua where elements of different chains are disjoint. The intersection of each of these $n$ chains is a continuum, and $f^{-1}(\bigcap \mathcal{L})$ is the union of these intersections. Therefore, $\mathcal{L}$ belongs to $\mathcal{C}$. It follows that $\mathcal{C}$ has a minimal element.

If $X$ and $Y$ are spaces, a map $f$ is said to be $k$-crisp if for every proper subcontinuum $L$ of $Y, f^{-1}(L)$ is the union of $k$ disjoint continua and $f$ restricted to each of the $k$ disjoint continua comprising $f^{-1}(L)$ is a homeomorphism onto $L$. Two elements of $X, x$ and $y$, are said to be siblings if $f(x)=f(y)$.

The following lemma extends a lemma of Griffus [3] to a more general class of spaces.

Lemma 2. Let $X$ be a space and let $Y$ be a continuum. If $f: X \rightarrow Y$ is a $k$-crisp map, then $f$ is locally one-to-one.

Proof. First, it is shown that $f$ maps every component $H$ of $X$ onto $Y$. Suppose that $f(H)$ is a proper subcontinuum of $M$. As a component of $X, H \subset f^{-1}(M)$ is a component of $f^{-1}(M)$. Since $f$ is $k$-crisp, $H$ is mapped onto $M$, which is a contradiction. It follows that $X$ has no more than $k$ components.

According to a lemma of Griffus [3], which is a generalization of a result of Mioduszewski [8, there exist nonempty open sets $U_{1}, U_{2}, \ldots, U_{k}$ in $X$ such that

(1) $\bar{U}_{i} \cap \bar{U}_{j}=\emptyset$ for every $i, j \in\{1,2, \ldots k\}$ such that $i \neq j$.

(2) $\left|f^{-1}(f(x)) \cap U_{i}\right|=1$ for every $x \in \bigcup_{j=1}^{k} U_{j}$ and every $i \in\{1,2, \ldots, k\}$.

(3) For every $i \in\{1,2, \ldots, k\}, f$ is locally one-to-one at each point of $\bar{U}_{i}$.

Let $U$ denote the set $\bigcup_{i=1}^{k} U_{i}$. Suppose that $f$ is not locally one-to-one at the point $z$. Let $C$ denote the component of $X$ containing $z$. There exist two disjoint sibling sequences $\left\{p_{i}\right\}$ and $\left\{\hat{p}_{i}\right\}$ in $C \backslash \bar{U}$ each of which converges to $z$.

For each $p_{i}$, let $C_{i}$ be the component of $p_{i}$ in $C \backslash U$. Since $C \backslash U$ is a nonempty, proper, closed subset of the continuum $C$, the Janiszewski lemma [9] implies that the continuum $C_{i}$ must bump the boundary of $C \cap U$. Let $x_{i}$ be an element of $C_{i} \cap \operatorname{Bd}(U)$. The $k$-crisp property implies that there exists a continuum $\hat{C}_{i}$ that is disjoint from $C_{i}$ and that contains $\hat{p}_{i}$. Since all siblings of $x_{i}$ are in the boundary of $U$, there is a sibling of $x_{i}, \hat{x}_{i}$ in $\hat{C}_{i} \cap \operatorname{Bd}(U)$.

There exists a common convergent subsequence of the sequence $\left\{C_{i}\right\}$ and of the sequence $\left\{\hat{C}_{i}\right\}$. Denote the limiting continuum of the common subsequence of $\left\{C_{i}\right\}$ by $A$ and the limiting continuum of the common subsequence of $\left\{\hat{C}_{i}\right\}$ by $B$. The continuum $A$ must contain the point $z$, as well as a limit point of the sequence $\left\{x_{i}\right\}$, call it $a$. The continuum $B$ must also contain the point $z$, as well as a limit point of the sequence $\left\{\hat{x}_{i}\right\}$, call it $b$. The points $a$ and $b$ must be distinct as well as siblings. However, this is a contradiction since $f(A \cup B) \neq Y$ and $f$ is $k$-crisp. Therefore, $f$ is locally one-to-one.

Theorem 3. No tree-like continuum is the confluent $k$-to-1 image of any continuum, for any $k \geq 2$.

Proof. Suppose that there exists a confluent $k$-to-1 map $f: X \rightarrow Y$ from a continuum $X$ onto a tree-like continuum $Y$, where $k \geq 2$. Choose $n$ to be the largest integer in the set $\{2,3, \ldots, k-1\}$ such that there is a continuum $M \subseteq Y$ whose inverse image has $n$ components. By Lemma 1, it may be assumed that $M$ is minimal. 
Since the property of being tree-like is hereditary, $M$ is tree-like. The restriction of $f$ to $f^{-1}(M)$ is $k$-crisp. Indeed, if $L$ is a proper subcontinuum of $M$, then $f^{-1}(L)$ has at least $k$ components. Since $f$ is confluent, each of them is mapped onto $L$. Hence $f^{-1}(L)$ has exactly $k$ components, on which $f$ is one-to-one.

By Lemma 2 the restriction of $f$ to $f^{-1}(M)$ is locally one-to-one. A theorem of J. Heath [6] states that every locally one-to-one map from a continuum onto a tree-like continuum is a homeomorphism. Therefore, $f$ restricted to any one of the $n$ components of $f^{-1}(M)$ is one-to-one. Hence, $f$ restricted to $f^{-1}(M)$ is $n$-to-one, which is a contradiction.

The final result follows from a result of $\mathrm{H}$. Cook [1], namely that any map from a continuum onto a hereditarily indecomposable continuum is confluent.

Corollary 4. No hereditarily indecomposable tree-like continuum is the continuous $k$-to-1 image of a continuum for any $k \geq 2$.

\section{REFERENCES}

1. H. Cook, Continua which admit only the identity map onto non-degenerate subcontinua, Fund. Math. 60 (1967), 241-249. MR 36:3315

2. W. H. Gottschalk, On k-to-1 transformations, Bull. Amer. Math. Soc. 53 (1947), 168-169. MR 8:481d

3. L. R. Griffus, Exactly k-to-1 maps between metric continua, Ph.D. thesis, Auburn University, 1996.

4. J. Heath, Tree-like continua and exactly k-to-1 functions, Proc. Amer. Math. Soc. 105 (1989), no. 3, 765-772. MR 89g:54028

5. 2 -to-1 maps with hereditarily indecomposable images, Proc. Amer. Math. Soc. 113 (1991), no. 3, 839-846. MR 92c:54012

6. Each locally one-to-one map from a continuum onto a tree-like continuum is a homeomorphism, Proc. Amer. Math. Soc. 124 (1996), no. 8, 2571-2573. MR 97c:54016

7. _ Exactly k-to-1 maps: from pathological functions with finitely many discontinuities to well-behaved covering maps, Continua with the Houston Problem Book, Lecture Notes in Pure and Applied Mathematics, vol. 170, Marcel Dekker, New York, 89-102. MR 96d:54015

8. J. Mioduszewski, On two-to-one continuous functions, Dissertationes Math. (Rozprawy Mat.) 24 (1961), 43 pp. MR 26:3021

9. Sam B. Nadler, Jr., Continuum theory: an introduction, Marcel Dekker, Inc., 1992. MR 93m:54002

10. S. B. Nadler, Jr. and L. E. Ward, Jr., Concerning exactly $(n, 1)$ images of continua, Proc. Amer. Math. Soc. 87 (1983), 351-354. MR 84c:54059

Department of Mathematics, University of West Alabama, Station 7, Livingston, Alabama 35470

E-mail address: teg@uwa.edu 